\title{
The similarity solution for turbulent mixing of two-layer stratified fluid
}

\author{
J. A. Whitehead
}

Received: 10 March 2008 / Accepted: 29 May 2008 / Published online: 19 June 2008

(C) The Author(s) 2008

\begin{abstract}
Experiments are reviewed in which a two-layer salt-stratified tank of water was mixed by turbulence. The density profile began as a single step and evolved to a smooth mixed profile. The turbulence was generated by many excursions of a horizontally moving vertical rod with Richardson number $R i>0.9$ and Reynolds Number $R e>600$. There was almost perfect collapse of all the profiles to one universal profile as a function of a similarity variable. We develop a theoretical model for a simple mixing law with a buoyancy flux that is a function of internal Richardson number Rii. A similarity equation is found. A flux law that increases with small Rii and decreases with large Rii is considered next. Since no analytical solution is known, the similarity concept is tested by numerically integrating the equations in space and time. With buoyancy flux monotonically increasing with internal Richardson number, the similarity approach is valid for a profile starting from a slightly smoothed step. However, a shock forms for a mixing law with higher initial Rii (so that buoyancy flux decreases with Richardson number) and the similarity approach is invalid for those initial conditions.
\end{abstract}

Keywords Turbulence $\cdot$ Mixing $\cdot$ Stratified $\cdot$ Similarity solution $\cdot$ Layered fluid

\section{Introduction}

This paper reviews some experimental results obtained by Ian Stevenson [1]. The work itself involves mixing by turbulence that is produced uniformly throughout all depths in layered water. The experiment is motivated by the fact that strong currents spontaneously develop near saddle points in the ocean floor [2,3]. They exhibit high values of shear and mixing which has renewed our interest in mixing within layered fluid. Many saddle points are distributed throughout all depths of the ocean because of the complex topography of ocean

\footnotetext{
J. A. Whitehead $(\varangle)$

Department of Physical Oceanography, MS\#21, Woods Hole Oceanographic Institution, Wood Hole, MA 02543, USA

e-mail: jwhitehead@whoi.edu
} 
ridges, therefore, the interest here is to have turbulence produced equally at all depths. The density field evolves as it is stirred by turbulence starting from two layers of water of differing salinity. There are two governing dimensionless parameters: a Reynolds number $\operatorname{Re}=U d / v$ for the turbulence, and either an initial Richardson number $R i=g \Delta \rho d / \rho_{0} U^{2}$ for layered fluid, or an internal and local Richardson number Rii $=N^{2} d^{2} / U^{2}$ for smoothly stratified fluid. In these formulas, the acceleration of gravity is $g, \Delta \rho$ is the initial density difference of two layers, $\rho$ is horizontally averaged density of the fluid, $\rho_{0}$ is its average throughout the depth of the tank, $d$ is the length scale of the turbulence source, $U$ is the speed of the turbulence source, $v$ is the viscosity of the fluid, and $N^{2}=-\left(g / \rho_{0}\right) d \rho / d z$ is local stratification frequency. Molecular diffusivity of the salt is neglected by assuming it is not of first order importance to the mixing dynamics if Reynolds number is high enough.

The experiment extends Linden's experiments [4] where a grid is dropped through two layers with differing salinity. The turbulence in the wake mixed the fluid. Buoyancy flux (gravity times density flux) was easily estimated by measuring the evolution of the density field. Here, we generate many such stirring events instead of only one and then we document the evolution of the density field using density profiles between successive stirring events. Using a similarity variable, the evolving density profile collapses to one profile. We then describe the implications in terms of a theoretical model in slightly more general terms than discussed previously. To our knowledge, this collapse for turbulent mixing is not previously reported.

\section{The experiment}

An acrylic tank that is $0.6 \mathrm{~m}$ high and with a horizontal cross-section $0.1 \mathrm{~m} \times 0.2 \mathrm{~m}$ was initially filled with approximately $0.25 \mathrm{~m}$ of salt water $(\mathrm{NaCl}$ solution, density $\rho$ varied in the 11 experiments from $1,030 \mathrm{mg} / \mathrm{m}^{3}$ to $1,200 \mathrm{~kg} / \mathrm{m}^{3}$ ). Then, an equal thickness of fresh water was carefully added using a float-mechanism. This procedure created two distinct layers of differing density with a sharp interface between them. Both layers were close to room temperature so that heat transfer effects between the layers could be neglected. A descending conductivity probe measured density profiles at a speed of $0.003 \mathrm{~m} / \mathrm{s}$. The sharpness of the initial density profiles of all the runs showed that this value of probe speed gave excellent fine-scale resolution of the profile. A mixing rod traversed back and forth along the wide direction across a $0.2 \mathrm{~m}$ long region, and the wake produced relatively uniform turbulence throughout the depth of the tank. The mixer was started after we recorded the initial density profile. After a certain number of rod excursions back and forth, mixing stopped and after a 2 min settling period, another profile was taken. Then, the same number of mixing excursions followed by a profile continued cyclically. A total of up to 300 rod excursions constituted an experiment. Rod diameter $d$ is the primary imposed length for the turbulence and therefore we take this to be the length scale for turbulence in this experiment. The values of $d$ used were $1.27,1.8$, and $2.68 \times 10^{-2} \mathrm{~m}$, and the range of the values of $U$ was $0.05-0.071 \mathrm{~m} / \mathrm{s}$. Using the values for reference density and viscosity at $20^{\circ} \mathrm{C}$, the 11 experimental runs had initial Richardson numbers ranging between $0.93<R i<4.84$ and Reynolds numbers extending between $638<R e<1765$.

At the beginning of a typical run, the measured profile almost exactly duplicates a step profile and because of the mixing subsequent profiles are more spread out. Correspondingly, the initial local values of $R i i$ (proportional to vertical derivative) are very large in the vicinity of the interface and virtually zero in each layer. After mixing starts, the maximum value of Rii at the interface decreases for each excursion, and Rii is measurable from the data throughout 


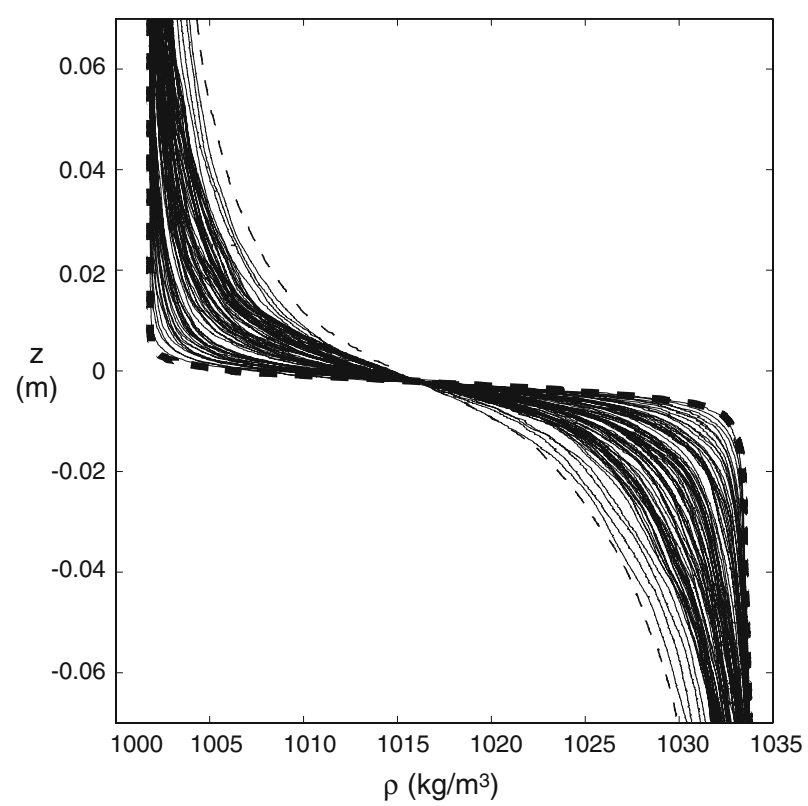

Fig. 1 Density profiles [1] throughout a typical run at equal mixing intervals (The first profile is a thick dashed curve and the last is a thin dashed curve.)

the fluid. A typical size of $R i i$ for each experiment can be estimated as $R i i \cong d R i / D$, where $D$ is water depth $(0.50 \mathrm{~m})$. The range for these experiments is $0.024<d R i / D<0.174$. These values caused us to expect that the experiment lies outside the range $R i i>1$ where layer formation was found in previous experiments $[5,6]$.

Each run produced about 80 profiles, with a typical set shown in Fig. 1. Drift of the calibration constants that convert voltage to density was inconsequential for the duration of all runs. Instrumental noise was small (of order $10^{-4}$ of the maximum range measured) but since an analysis of buoyancy flux required calculation of vertical derivative and the subtraction of two records (see next section), the scatter of the differences between closely spaced points or sequential profiles was considerable. The density values had precision better than $0.1 \%$. An error also arises from small drifts in the position of the probe. To account for these errors we centered the profiles by adding a small uniform shift, a common practice in these types of experiments $[1,4-6]$. The centered profiles were averaged over 5 points taken at $0.001 \mathrm{~m}$ intervals to smooth noise, and the centered density profiles yielded values of density as a function of depth, from which we calculated buoyancy frequency and from subsequent profiles determined buoyancy flux and change in potential energy.

In the experiment, time is taken to be linearly proportional to the number of excursions. All our laboratory experiments showed strikingly good data collapse using the similarity variable of depth divided by the square root of the number of excursions $n$. The collapse for actual data is remarkable (Figs. 2 and 3). Thirteen runs in all exhibited similar collapse, although not all are as good as these examples (probably from experimental effects such as not exactly equal layer depths, or slight effects from room temperature influences, for example). Stevenson found that the first few profiles had apparently not yet adjusted to the exact experimental similarity profile shape. Because of this, the best collapse excluded the first few 

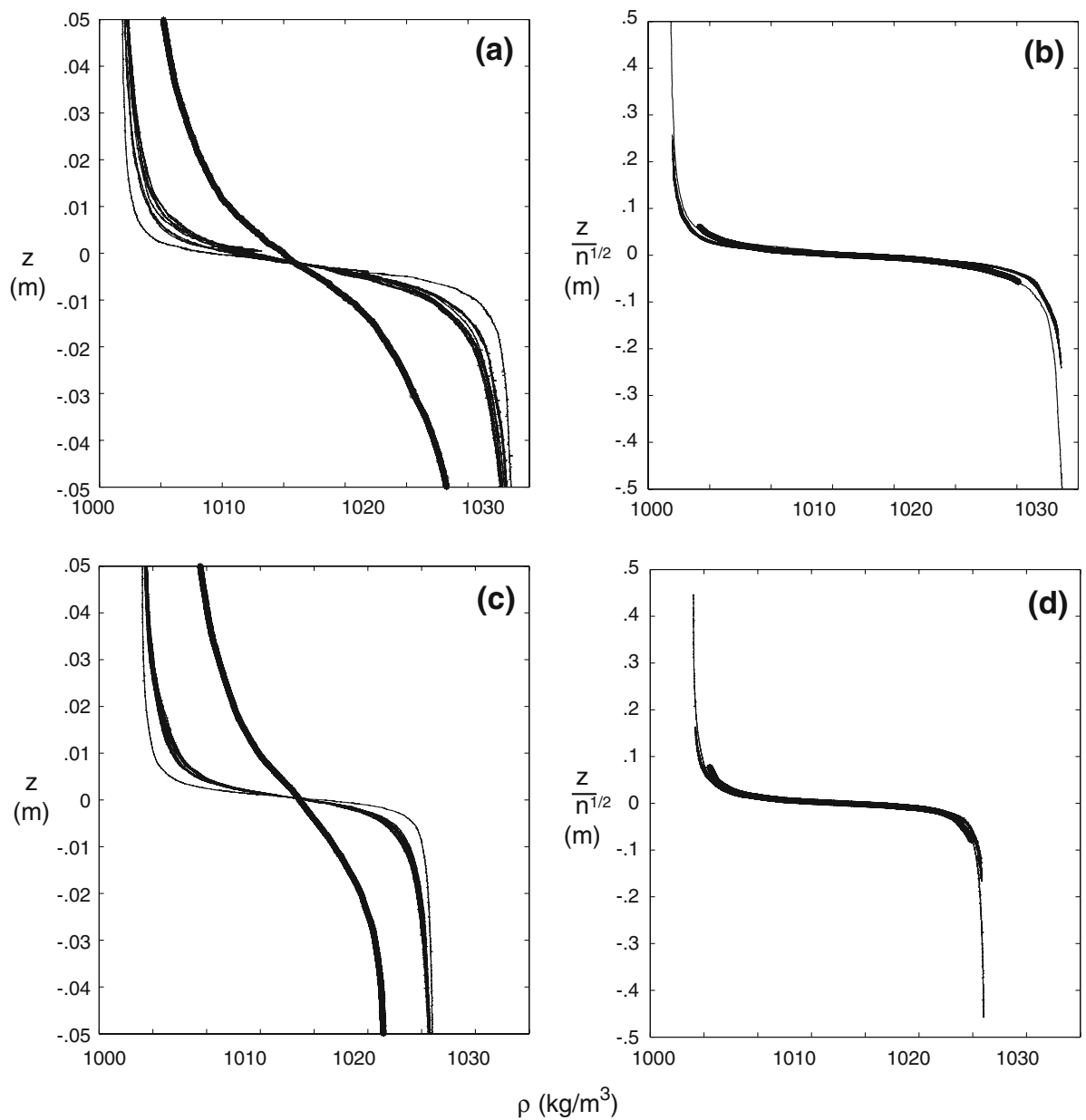

Fig. 2 Selected laboratory experimental profiles (left) and their collapse [1] when divided by the square root of the number of traverses (right). The traverse numbers for panels (a) and (b) are: 20 (thin line); 41-84 (cluster of lines); 300 (thick line). The traverse numbers for panels (c) and (d) are: 14 (thin line); $40-60$ (cluster of lines); and 336 (thick line)

profiles. We also saw poorer fit to a perfect collapse for some of the final profiles. Possibly those later profiles exhibited effects from the top and bottom boundaries of the chamber.

\section{A simple model}

The conservation of density obeys

$$
\frac{\partial \rho}{\partial t}=-\frac{\partial F_{d}}{\partial z}
$$

with $F_{d}$ the density flux in the $z$ direction, and $z$ is positive upward. Whitehead and Stevenson, show results for specific models of $F_{d}$. Here we illustrate the general consequences of turbulence driven by a source with velocity and length scales that are independent of depth. 
Fig. 3 Close-up of traverses number 20 (black) and 300 (grey) with depth divided by $\sqrt{n}$

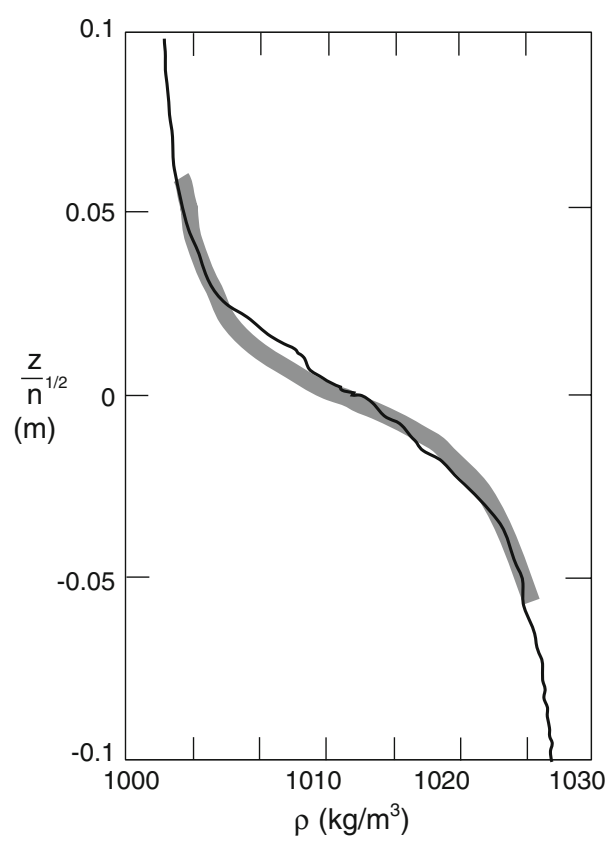

Taking a vertical derivative,

$$
\frac{\partial^{2} \rho}{\partial t \partial z}=-\frac{\partial^{2} F_{d}}{\partial z^{2}}
$$

and multiplying by $g / \rho_{0}$ the equation is

$$
\frac{\partial N^{2}}{\partial t}=\frac{g}{\rho_{0}} \frac{\partial^{2}}{\partial z^{2}} F_{d} .
$$

Next, let $F_{d}=\frac{\rho_{0}}{g} U d F$ (Rii) and make the equation non-dimensional using the time scale $d / U$ and vertical length scale $d$. The result is

$$
\frac{\partial R i i}{\partial t^{\prime}}=\frac{\partial^{2}}{\partial z^{\prime 2}} F(R i i) .
$$

This equation can be converted to an ordinary differential equation using the similarity variable $\eta=\frac{z^{\prime}}{2 \sqrt{t^{\prime}}}$ and the governing equation becomes

$$
\eta \frac{d}{d \eta} R i i=-\frac{1}{2} \frac{d^{2}}{d \eta^{2}} F(R i i)
$$

We don't know of any analytical solutions for (3.5), but the error function is a well-known solution for (3.1) with such a similarity variable. It is found for the special case of $F_{d}=-\kappa \frac{\partial \rho}{\partial z}$ (the diffusion equation) with the initial condition of two overlying uniform layers of different density. Equation (3.5) will not have such an initial condition in general for the case of two layers since it applies to a vertical derivative of density rather than to density itself. In spite of this, numerical calculations [1] have found an example for Eq. 3.4 that collapsed to such a similarity variable using the function $F(R i i)=D \frac{R i i}{1+R i i^{2}}$. The variable $D$ is an equivalent diffusion for this nonlinear flux law. Those previous calculations started with two overlying 

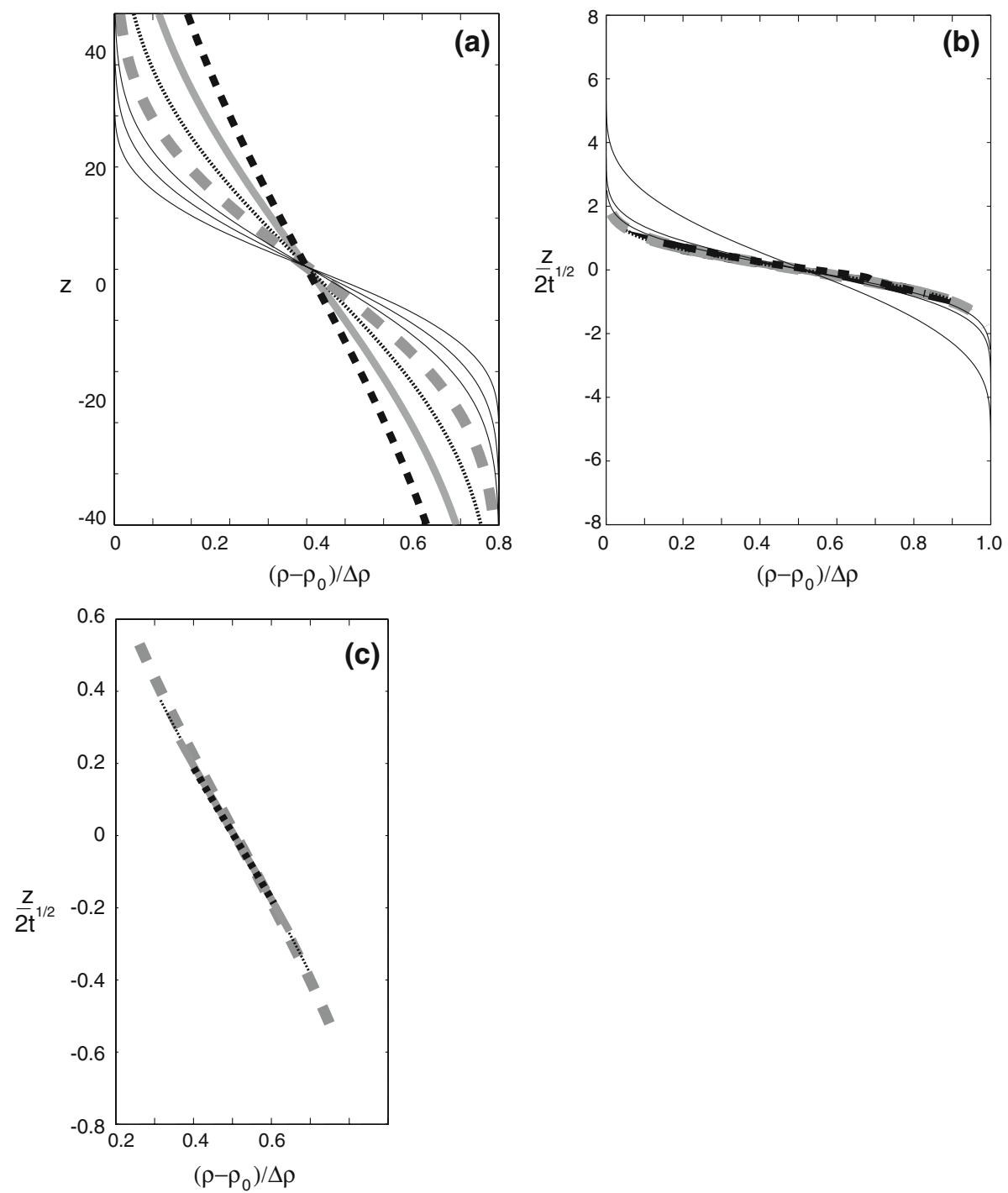

Fig. 4 Theoretical density profiles with $D=0.01$ for various dimensionless times $(10,50,100,200$ (long dashed grey), 400 (thin black dashed), 800 (gray) and 1600 (thick black dashed). (a) Profiles versus depth. (b) The same profiles plotted with the similarity variable. (c) Close-up for the four longest times plotted with the similarity variable. These overlap significantly

layers of 50 grid points each with an initial region of 10 grid points with smoothed density change between the two layers.

Although the results verified the similarity scaling for small $D$, results for $D>1$ raised a question concerning the robustness of the similarity scaling. To be specific, it was mentioned that there was a breakdown to a shock for $D>1$. Unfortunately their numerical calculation had only 100 levels and no details of the shock formation were possible.

To determine more details of the shock, additional calculations have now been done with a higher resolution for large $D$. The domain has 1,000 vertical points. The initial condition 

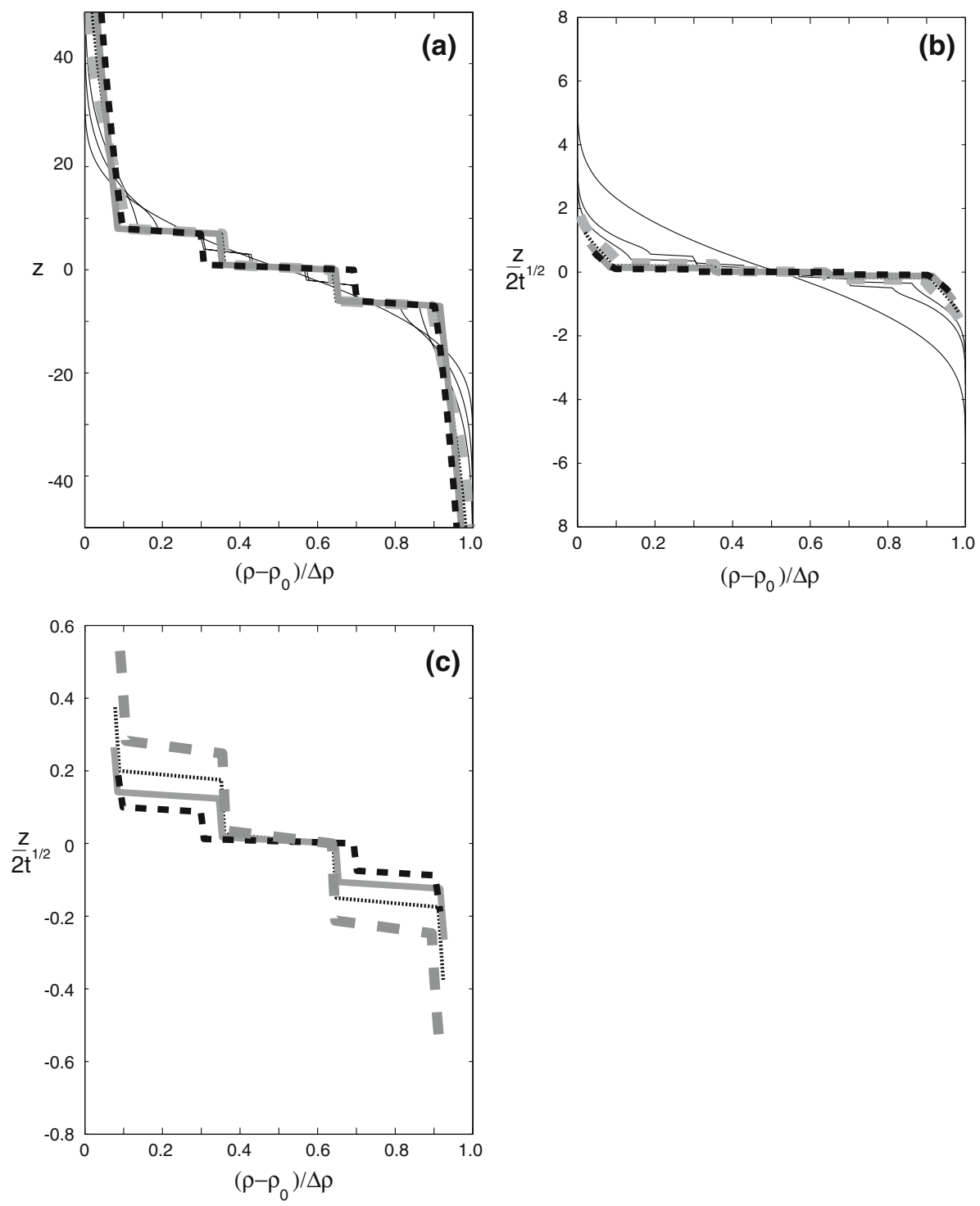

Fig. 5 Theoretical density profiles with $D=2.0$ for various dimensionless times. The dimensionless times and panels are the same as in Fig. 2

is $\operatorname{Rii}(z, t=0)=\delta(z)$ where $\delta$ is a linearly changing distribution of stratification (with a peak in the middle) spread over the central 50 grid points. First, the case with $D=0.01$ shows that, after an initial period, the distribution (Fig. 4a) settles down to agreement with similarity theory (Fig. 4b,c).

In contrast, for $D=2$ the calculations do not verify a similarity theory. Instead, a shock forms near mid-depth and within the shock are steps that remain frozen for long periods of time. Figure 5 shows results for the same parameters as in the panels of Fig. 4 except that $D=2.0$. The collapse of profiles is better in real time than with scaled time. 
Fig. 6 Comparison of calculations and measurements. A similarity error function is shown by large black dashes, the numerical similarity calculation is a wide gray curve, and the experiment curves are the same as in Fig. 2b

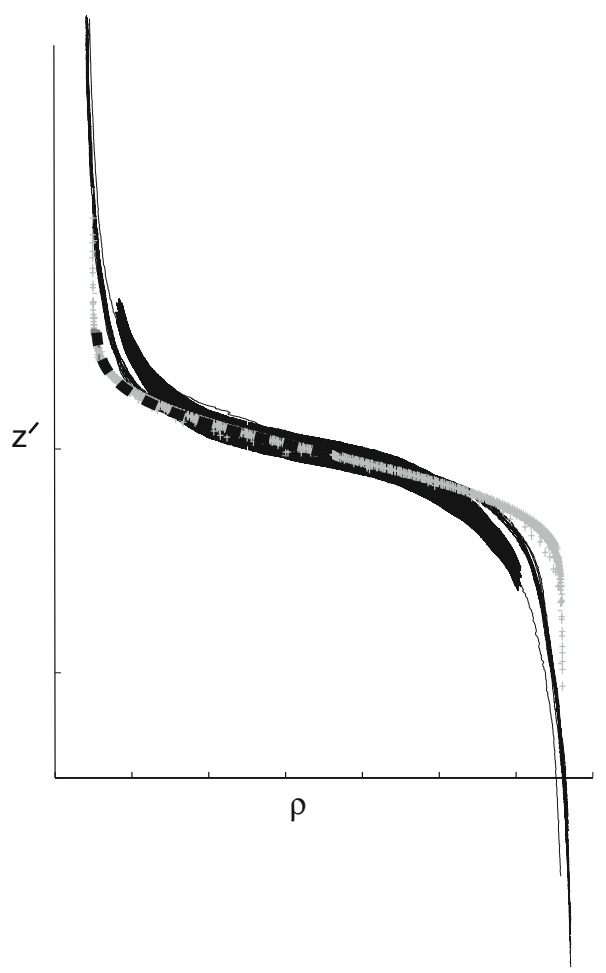

\section{Application of the similarity scaling to data}

Next, we compared theory and data [1] by overlaying the collapsed theoretical similarity curve in Fig. 4b and the collapsed data in Fig. 2b. To make the best fit, the depth for the theoretical curve was stretched to have the same slope as the experimental data at mid-depth (Fig. 6). The theory has moderately good agreement with the data but certainly the fit is not perfect. The theory has more curvature away from the mid-point than the data. Therefore, the theoretical curve is not the same as the experiment in detail.

Using the similarity variable, the thickness of the transition region between the upper and lower layer in Fig. 2 can be used to infer a value of eddy diffusivity. In dimensional units, the similarity variable is $\eta=z / 2 \sqrt{d U t}$ so that $U d$ serves as a substitute for the diffusivity. Results show that the region with $90 \%$ of the change between the upper and lower layer is found within about $\eta= \pm 0.05$. Let us compare this to the complementary error function. In that case, we can define the transition layer to lie within about $\eta= \pm 1$. For our present experiment to have the same dimensionless thickness, we should use the similarity variable $\eta=z / 2 \sqrt{0.05^{2} d U t}$. In other words, the experiment shows that the effective diffusivity is about $0.05^{2} U d=2.5 \times 10^{-3} U d$. This is somewhat smaller than the value $\kappa_{e f f}=6.7 \times 10^{-3} U_{0} M$ found by Holford and Linden [5] where $M$ is the grid spacing. Since there is not a direct geometrical correspondence between the rod used in this experiment and the grids used by Holford and Linden, our two results agree as well as can be expected. 

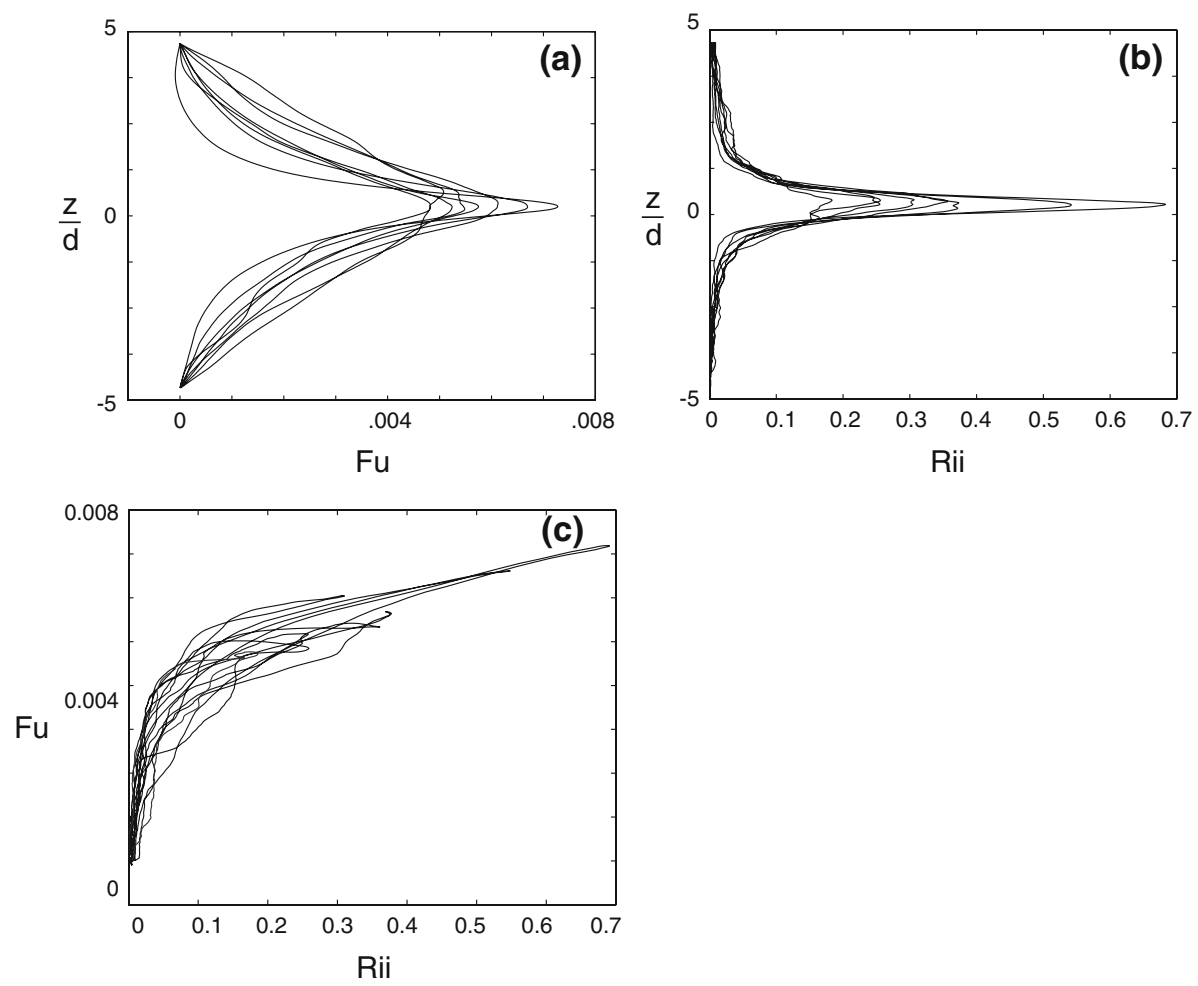

Fig. 7 (a) Measured flux as a function of depth. The flux is not total flux (see text). (b) Flux as a function of Richardson number, and (c) flux versus Richardson Number Rii from one experimental run. Note that $F_{u}$ monotonically increases with Rii

\section{Discussion}

Buoyancy flux and potential energy evolution were also measured but results are incomplete since the probe was unable to traverse the entire depth of the tank. Buoyancy flux $F_{u}$ was determined by integrating Eq. 1 from the bottom of the measurement region to find $F_{d}$ and then multiplying by gravity $g$. Thus, it is not the total buoyancy flux through the region, but only a deviation from the flux that comes from the unmeasured deep part of the tank. Generally, the measurement (Fig. 5) showed a monotonic increase of buoyancy flux with Rii, and no decrease in flux at large Rii. The lack of fine structure in these experiments and the numerical model is thus consistent with the regime diagram of Holford and Linden [3], who report no layers in that range of Rii. Finally, estimates of the amount of turbulent kinetic energy going to mixing processes show that a maximum of approximately $5 \%$ of the kinetic energy generation rate by the moving rod noise.

No experiments have yet recovered the formation of a shock that is found in the numerical calculations in which $F$ attains a peak value with an increasing Rii and which then decreases for greater value. Moreover, we do not observe any evidence of such a decrease in flux when plotting flux versus stratification from the data. Unfortunately, those measurements are not definitive because the profiles did not extend to the very bottom and top of the tank. In addition, the values of Rii are not large enough to produce layering that is seen in experiments with smooth profiles $[5,6]$. 


\section{Conclusions}

The application of similarity variables to the data from stratified turbulent mixing shows excellent collapse over a wide range of times. Specifically, the collapse of data when depth is divided by the square root of the number of excursions is remarkably good over a span of starting at excursion number 8 and extending to excursion 300 . The similarity approach here has been developed subject to the simplification that flux is a monotonically increasing function of Rii alone. These experimental results differ from the results of Holford and Linden [5], who concluded that their experiment required an additional dimensionless number. Finally, this laboratory observation of such a similarity-based approach to turbulently mixed data gives support to the use of scaling in oceanography [7]. The experiment obtained reasonable results but quantitative precision of flux laws was not obtained because the density was not sampled throughout the entire depth of the tank.

Acknowledgements Ian Stevenson was supported as a summer student fellow of Woods Hole Oceanographic Institution. The laboratory expenses were supported by WHOI's Ocean and Climate Change Institute.

Open Access This article is distributed under the terms of the Creative Commons Attribution Noncommercial License which permits any noncommercial use, distribution, and reproduction in any medium, provided the original author(s) and source are credited.

\section{References}

1. Whitehead JA, Stevenson I (2007) Turbulent mixing of two-layer stratified fluid. Phys Fluids 19:125104. doi:10.1063/1.2821913

2. Whitehead JA (1989) Internal hydraulic control in rotating fluids — applications to oceans. Geophys Astrophys Fluid Dyn 48:169-192. doi:10.1080/03091928908219532

3. Thurnherr AM, St. Laurent LC, Speer KG, Toole JM, Ledwell JR (2005) Mixing associated with sills in a canyon on the midocean ridge flank. J Phys Oceanogr 35:1370-1381. doi:10.1175/JPO2773.1

4. Linden PF (1980) Mixing across a density interface produced by grid turbulence. J Fluid Mech 100:691703. doi:10.1017/S002211208000136X

5. Holford JM, Linden PF (1999) Turbulent mixing in a stratified fluid. Dyn Atmos Oceans 30:173-198. doi:10.1016/S0377-0265(99)00025-1

6. Park Y-G, Whitehead JA, Gnanadesikan A (1994) Turbulent mixing in stratified fluids: layer formation and energetics. J Fluid Mech 279:279-311. doi:10.1017/S0022112094003915

7. Ledwell JR, Watson AJ, Law CS (1998) Mixing of a tracer in the pycnocline. J Geophys Res O 103:21499_ 21529. doi:10.1029/98JC01738 